\title{
ASH 2011: "Real generosity toward the future lies in giving all to the present"
}

\author{
Michael Steurer
}

Received: 15 July 2012 / Accepted: 25 July 2012 / Published online: 13 September 2012 (C) Springer-Verlag Wien 2012

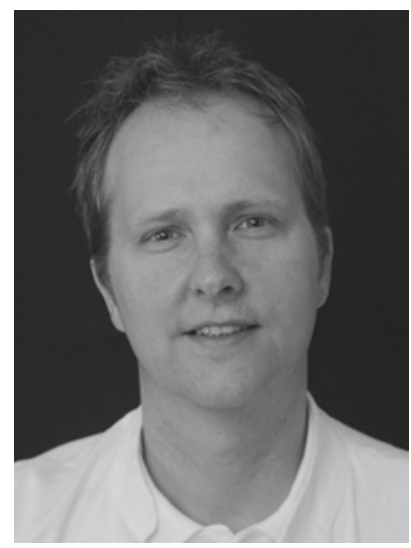

Michael Steurer
The 53rd annual meeting of the American Society of Hematology (ASH), which took place on December 10th-13th, 2011 in San Diego, California was attended by more than 20,000 participants. More than 3,000 presentations comprised the data from out of more than 5,000 abstracts. Extracting the most relevant data out of this wealth of information is certainly a difficult task. Therefore, in this issue of MEMO, distinct experts in the field of haematology have taken on the task of working through the data to present-of course from a subjective point of view-a selection of the most interesting and clinically relevant data for clinical decision making in the future. As in the past 2 years, the overall emphasis at ASH 2011 was not on large randomized clinical trials aimed at definitely determining the role of defined treatments but rather on small hypothesis-generating translational studies.

Probably, the most relevant practice-changing progress is currently being made in the field of indolent lymphoma and chronic lymphocytic leukaemia. Recent insights into the complex interplay between the neoplastic clone and the microenvironment has led to the discovery of new pathogenetic pathways and ultimately to the development of a variety of new targeted drugs. As discussed in the CLL and indolent lymphoma section of this issue, preliminary results from pilot trials incorporating these novel treatment strategies into clinical practice are very encouraging. Compounds such as the Bruton's tyrosine kinase inhibitor ibrutinib (formerly PCI-32765) or the phosphatidylinositol-3-kinase inhibitor CAL 101

\footnotetext{
M. Steurer, MD ( $\bowtie)$

Department of Internal Medicine V, Laboratory for Molecular Genetics \& Diagnostics, Innsbruck Medical University, Anichstr. 35, 6020 Innsbruck, Austria

e-mail: michael.steurer@i-med.ac.at
}

are only the first representatives of a new class of drugs that will definitely have great impact on future therapies.

But, also in the field of T-cell lymphomas, a long and still neglected field of haematology, significant progress is being made. As discussed by Hopfinger et al., two new drugs have recently been approved by the United States Federal Drug Administration (FDA). These are the first drugs that are approved specifically for the treatment of T-cell lymphoma, at all. Pralatrexate, a folate inhibitor and the histone deacetylase inhibitor romidepsine may both achieve response rates of approximately $25 \%$ when given as a single drug. Thus, they represent attractive compounds to be administered together with conventional chemotherapy regimens such as $\mathrm{CHO}(\mathrm{E}) \mathrm{P}$ or Da-EPOCH \pm autologous stem cell transplantation. Moreover, the monoclonal anti-CD30 antibody brentuximab vedotin, which is conjugated with the tubulin polymerization inhibitor auristatin, may be able to significantly improve the outcome of CD30-positive T-cell lymphomas, in particular anaplastic large cell lymphomas.

With its impressive activity, brentuximab vedotin will also play an important role in the treatment of relapsed/ refractory Hodgkin's lymphoma and it is probably only a matter of time until this new drug will be incorporated into first-line treatment, e.g. for advanced disease stages. However and as discussed by Willenbacher and Willenbacher, currently available treatment modalities still leave room for improvement. Through systematic reduction of chemotherapy and radiotherapy doses in the framework of well-designed clinical trials, e.g. by restricting consolidating radiotherapy only to patients with PET-positive nodes as in the German HD-15 trial, clinical results may be further improved as severe side effects such as the occurrence of secondary primary neoplasms can be significantly reduced. 
In the field of acute myeloid leukaemias (AML), recent years have taught us new lessons on the genetic complexity of these diseases. However and as discussed by Johannes Clausen, the true impact of these data on clinical management is still under investigation. A major progress, however, has been made through the re-discovery of the anti-CD33 immunotoxin gemtuzumab ozogamicin (GO). After several AML study groups have systematically re-explored the activity of GO for AML treatment and re-established the clinical value of this compound, e.g. by demonstrating a 10 months survival benefit for older AML patients (ALPHA 0701 trial). These results are also a good example of how important it is to maintain and support independent clinical research projects carried out by non-profit academic institutions as the manufacturer, discouraged from (seemingly) negative early-trial results, had already withdrawn GO from the market in 2010. In view of the latest trial results, however, a renaissance of GO seems very likely.

Concerning myelodysplastic syndromes (MDS), considerable progress is being made in the field of basic MDS biology, in particular molecular mechanisms. As discussed by Pfeilstöcker and Stauder, the discovery of a completely new mechanism of leukaemogenesis through defects in the splicing machinery sheds light into the pathogenesis not only of MDS but also a whole range of haematologic disorders. Moreover, these findings will probably also have major implications for future patient care in terms of diagnosis, assessment of prognosis and as a druggable target. Concerning MDS therapy today, various trials are currently exploring different combinations of recently discovered drugs that are active in MDS such as lenalidomide, thrombopoietin mimetics, azanucleosides and histone deacetylase inhibitors. However, none of the combinations have reached the status of a clinical standard as yet.

At present, clinical research in the field of chronic myeloid leukaemia (CML) concentrates on the optimal use of the second- and third-generation tyrosine kinase inhibitors. As discussed by Stefan Schmidt, nilotinib and dasatinib both offer the possibility of reaching fast and deep molecular responses. However, the true clinical impact of these findings is still a matter of debate and more data is needed to ultimately define the role of these drugs in the treatment algorithm of CML.

In summary, progress in the field of haematology is going on with perplexing speed. It is exciting to witness how novel treatment concepts and new drugs arise and make their way from the bench to the bedside. These results should give the haematologic community further motivation for the conduct of translational research and clinical trials in order to improve future patient care. Or in the words of Albert Camus: "Real generosity toward the future lies in giving all to the present."

\section{Conflict of interest}

The author has no conflict of interest to disclose. 